\title{
Understanding the effect of information presentation order and orientation on information search and treatment evaluation
}

\section{Claire Louise Heard (MSc)}

(Corresponding author)

Department of Psychology, King's College London, Guy’s Campus, SE1 1UL, London, United Kingdom, claire.1.heard@kcl.ac.uk, 02078481298

\section{Tim Rakow (PhD)}

Department of Psychology, King's College London, Guy’s Campus, SE1 1UL, London, United Kingdom, tim.rakow @kcl.ac.uk, 02078486228

\section{Tom Foulsham (PhD)}

Department of Psychology, University of Essex, Wivenhoe Park. Colchester, CO4 3SQ, United Kingdom, foulsham@essex.ac.uk, 01206874159

This work was conducted at both the University of Essex and King's College London. It has been presented at the 2016 JDMx meeting for Early-Career Researchers (oral presentation); and at CogSci2017 (poster and refereed conference proceedings). These studies were funded by the PhD studentship awarded to the first author from the University of Cambridge (Winton Fund) and Economic Social Research Council-ESRC). The funding source had no role in the study topic, design, analysis or reporting. 
Role of order on search and treatment perceptions

\title{
Understanding the effect of information presentation order and orientation on information search and treatment evaluation
}

\begin{abstract}
$\underline{\text { Abstract }}$
Background: Past research finds that treatment evaluations are more negative when risks are presented after benefits. This study investigates this order effect: manipulating tabular orientation and order of risk/benefit information and examining information search order and gaze duration via eye-tracking.
\end{abstract}

Design: 108 (Study 1) and 44 (Study 2) participants viewed information about treatment risks and benefits, either in a horizontal (Left-Right) or a vertical (Above-Below) orientation, with the benefits or risks presented first (left side or at top). For four scenarios, participants answered six treatment evaluation questions (1-7 scales) that were combined into overall evaluation scores. Additionally, Study 2 collected eye-tracking data during the benefit/risk presentation.

Results: Participants tended to read one set of information (i.e., all risks or all benefits) before transitioning to the other. Analysis of order of fixations showed this tendency was stronger in the vertical (mean standardised mean rank difference further from $0, \mathrm{M}= \pm .88$ ) than horizontal orientation $(\mathrm{M}= \pm 0.71)$. Approximately $50 \%$ of time was spent reading benefits when benefits shown first but reduced to $\sim 40 \%$ when risks presented first (regression coefficient: $\mathrm{B}=-4.52, \mathrm{p}<.001)$. Eye-tracking measures did not strongly predict treatment evaluations, although time percentage reading benefits positively predicted evaluation when holding other variables constant $(\mathrm{B}=0.02, \mathrm{p}=.023)$.

Conclusion: These results highlight the impact of seemingly arbitrary design choices on inspection order. For instance, presenting risks where they will be seen first leads to relatively less time spent considering treatment benefits. Other research suggests these 
changes to inspection order can influence multi-option/multi-attribute choices and represents an area for future research.

\section{$\underline{\text { Introduction }}$}

When making health choices, an individual often learns about benefits and risks. Tabular formats, with risk and benefit information separated into clear columns or rows, are a common way of presenting such information in leaflets and websites. For example, a prostate cancer screening test fact sheet includes a table showing the benefits and risks of a test[1].

Although presented together, the reader does not apprehend all this information simultaneously. Rather, they must work their way through the table. Importantly, tables may differ in their orientation (presenting information sets horizontally 'side-by-side' or vertically 'above-and-below') and order of presentation (which cell contains each piece of information). To understand what these differences might mean for processing that information and for subsequent treatment choices and evaluations, we consider research on information search, spatial layout, and attention. This includes experiments that manipulate the order of information by controlling the sequence of presentation.

People usually examine information in an order consistent with their reading system. In most Western cultures, this means examining the top left of the page or screen first. Concordantly, eye-tracking reveals a bias in attention and search to the left side of space, with the first saccade typically made to the left of an image[2]; and that people spend twice as much time looking at the left side of a webpage[3]. Relatedly, horizontal saccades are made more frequently than vertical saccades when viewing images[4], which indicates that some orientations might lead to different orders of inspection. 
Experimental psychologists have manipulated the presentation order of information to understand whether, and how, this affects memory and decision making - with the "primacy" and "recency" of information being a key interest. In decision research, a primacy effect is when preferences or evaluations align more strongly with the valence of the first items of information that were encountered; while in recency effects, the attribute values presented last are better predictors of choice or evaluation[5]. Bergus, Levin and Elstein[6] manipulated information order for health-based information providing information about the risks and benefits to their participants. In a physician's waiting room, participants provided ratings of treatment favourability before and after learning about the risks and benefits of aspirin treatment ${ }^{1}$. The results showed a recency effect: those who learned about the benefits after the risks rated the treatment more favourably than those who learned about the risks after the benefits ${ }^{2}$, and were more than twice as likely to consent to treatment. A similar recency effect was found in a randomised trial of women at high risk of breast cancer shown the benefits and risks of tamoxifen[7]. Thus, treatment evaluations appear disproportionally influenced by the last piece of information processed, potentially because when the evaluation is made, the information presented last is more accessible in memory. This is consistent with some research on decision and evaluation tasks that involve sequential search though items of information[8][9][10]. In sum, simply presenting the same information about treatment risks and benefits in a different order may change how people perceive the treatment.

The current investigation examines two aspects of tabular layout: table orientation (whether categories of information are presented by rows or by columns), and information

\footnotetext{
${ }^{1}$ The benefit was that the risk of stroke was decreased with the use of aspirin. The risk information identified four potential side effects: 1) gastrointestinal problems, 2) ulcers, 3) bleeding problems, mostly minor, and 4) intracerebral haemorrhage.

${ }^{2}$ For treatment favourability rated on a 100-point scale: In the risks after benefits condition, favourability dropped by 10.9; for the benefits after risks condition, favourability dropped by $5.2(\mathrm{p}=.02)$.
} 
Role of order on search and treatment perceptions

order (which row/column displays a given piece of information). Our investigation extends previous research on order effects in patient decisions in two ways. First, in two studies, we investigate whether the recency effect found in previous studies of decision making[6] replicates when the risk and benefit information is presented in a table with risk and benefit information blocked into either separate columns or separate rows. Thus, we examine whether the recency effect found by Bergus et al.[6] also occurs when people follow their "natural" order of reading rather than being "fed" information one piece at a time. Because Bergus et al.[6] only found an order effect for a low-risk treatment, we restrict ourselves to examining such low-risk scenarios.

Second, we use eye-tracking (Study 2) to record the order in which people search through a table of treatment information. Previous studies, outside the medical domain, illustrate the value of such process-tracing approaches[11]. For example, Glöckner and Herbold[12] showed how recording eye-fixations can shed light on the strategies that people use to collect information prior to choosing between risky options. Based on the gaze-bias literature, we predict that items positioned top and left are more likely to be read before items positioned bottom and right. From the eye-gaze recordings, we also compute the number of transitions between benefits and risk. Because horizontal saccades are more common than vertical saccades, in reading but also in other tasks, we predict that in the horizontal condition, where risk and benefit information is presented side by side, switching between information sets will be higher. Conversely, when presented vertically with one information block presented above the other information block, reading order should be more constrained than when each information block is presented side-by-side, because participants will switch less often. We calculate the proportion of time spent examining benefits (vs. risks) to determine whether this is influenced by manipulating the orientation and position of 
information; and whether this, together with the other eye-tracking measures, predicts subsequent treatment evaluations.

\section{$\underline{\text { Methods }}$}

\section{$\underline{\text { Participants }}$}

There were 108 participants in Study 1 (treatment evaluations) and 44 in Study 2 (treatment evaluations plus eye-tracking). All participants were University of Essex students (undergraduates and postgraduates from a range of departments) who were over 16 years of age and gave written consent. Ethical approval was granted by the University's Research Ethics Committee. Participants were paid for their time, and completed a pre-screen questionnaire which provides the source for our demographic data. In Study 2, a minority $(<5 \%)$ of participants were excluded due to poor eye-tracking data quality, leaving the 44 participants reported above.

\section{$\underline{\text { Apparatus }}$}

In Study 2, an EyeLink 1000 eye-tracker and associated software package (Experiment Builder) were used to control the experiment and collect eye-tracking measures. The EyeLink 1000 has an average error of less than $0.5^{\circ}$ of visual angle.

\section{Materials \& Procedure}

Each participant was presented with four hypothetical scenarios embedded in a computercontrolled survey: aspirin for mild carotid stenosis, statins for high cholesterol, ACE (angiotensin converting enzyme) inhibitors for high blood pressure, and anticoagulant medicine for deep vein thrombosis. These four scenarios were chosen to fit with our design restriction to 'low risk' treatments. 
For each scenario (reproduced in full in Appendix 1), participants read about the situation (hypothetical, from the participant's perspective) which led to a medical diagnosis, the diagnosis itself, and the treatment to be considered. Next, on a separate screen, participants saw three risks and three benefits associated with that treatment. ${ }^{3}$ These were presented in one of two orientations. In the horizontal orientation, the separate lists of risks and benefits were displayed side-by-side (figure 1a). In the vertical orientation (figure 1b), information was presented with one set at the top of the screen and the other set underneath. As convenient terminology, which reflects the gaze-bias literature, we describe information presented left in the horizontal orientation and information presented at the top of the screen in the vertical orientation as being "presented first". For example, presenting "benefits then risks" means presenting the benefits on the left in the horizontal orientation, or presenting benefits above the risks in the vertical orientation.

\section{$* * *$ Figure $1 * * *$}

Participants read the risk/benefits and then pressed the space bar to advance to the next screen. Participants then answered six 7-point Likert scale evaluation questions for the treatment. Three were positively phrased and three negatively phrased. For example, one of the positively phrased questions asked: would you recommend this treatment (definitely no to definitely yes). A full list of all six questions is presented in Appendix 2. Each question was presented on a separate page and the screen advanced to the next question when a participant made their rating (by pressing the 1-7 keys). Participants could not return to a previous question or return to the benefit/risks information while making these responses and were told this at the start of the experiment. After answering all questions, participants saw the next scenario.

\footnotetext{
${ }^{3}$ By design, for each scenario, the number of words (or characters) was similar for risks and benefits.
} 


\section{General Design and Data Treatment}

All participants saw all four scenarios described above; with exactly the same information. Information layout was varied according to our manipulations of orientation and order of information. Both experiments used a $2 \times 2$ between-subjects design with two factors: orientation (Horizontal vs. Vertical), and order (Benefits then Risks vs. Risks then Benefits). In study 1, a Latin-Square design was used such that every participant saw each scenario in a different format. In study 2, assignment to one of these four formats was randomised for each scenario for each participant. Therefore, in study 1, each participant encountered each format once; while in study 2 , randomisation meant that a participant might not encounter every format and could encounter a format twice or more.

An overall treatment evaluation score was created that averaged the responses from the 6 Likert-scale ratings, after reverse coding the three negative items. This combined score was justified by very good scale reliability (Cronbach's alpha values for all scenarios: $\alpha-$ range $.87-.92$ in study $1 ; \alpha$-range $.85-.92$ in study 2 ). Thus, while conceptually the six questions reflected slightly different judgements, empirically these components were generally too strongly related to make separate analyses worthwhile. To illustrate, the median correlation between items for each scenario in Study 1/Study 2 were: $r=0.56 / 0.71$ (Aspirin), $\mathrm{r}=0.61 / 0.66$ (ACE Inhibitors), $\mathrm{r}=0.58 / 0.56$ (Anticoagulants) and r=0.56/0.44 (Statins).

\section{Eye-tracking variables and data treatment (Study 2 only)}

The eye-tracker recorded the location, order and duration of participants' fixations for each scenario. Separate interest areas (IAs) were created for risks and benefits from the union of areas around the title and three pieces of information. The total area for each IA subtended approximately $14.65^{\circ} \mathrm{X} 10.82^{\circ}$. We recorded the overall time spent attending to each IA, 
and the number of fixations in each IA. Fixations falling outside of these IAs were not analysed.

Raw data were screened and then transformed into measures reflecting the order and amount of attention given to risks and benefits. Each measure is described below.

SMRD Order: We used the standardised median rank difference (SMRD) score to generate a single score from ' -1 ' to ' +1 ', reflecting the order of in which information items (risks and benefits) were inspected. This score reflects the extent to which participants looked at benefits or risks first. Looking mainly at benefits before risks leads to a negative SMRD score, and if someone looks exclusively at benefits before transitioning to look at risks (without returning to look at benefits) their score is ' -1 '. Looking mainly at the risks first leads to a positive SMRD score. We adapted the formula for SMRD reported by Johnson, Häubl and Keinan[14] and others[15]:

$$
\frac{2\left(M R_{\text {Benefit }}-M R_{\text {Risk }}\right)}{\text { Number of Fixations }}
$$

where MR represents the median rank of the eye-fixation set. To calculate the two median rank scores, one for the benefit information $\left(\mathrm{MR}_{\text {Benefit }}\right)$ and one for the risk information ( $M R_{\text {Risk}}$ ), fixations were ranked from the first fixation (coded ' 1 ') to the last; then, $M R_{\text {Benefit }}$ and $\mathrm{MR}_{\text {Risk }}$ were computed. Taking a simplified example: If a person made 6 fixations BBRRBR - then the MR for benefits (B) would be 2 (the median of $1^{\text {st }}, 2^{\text {nd }}$, and $5^{\text {th }}$ positions) and the MR for risks would be 4 (the median of $3^{\text {rd }}, 4^{\text {th }}$ and $6^{\text {th }}$ positions). With these, the SMRD score would be: $[2 \times(2-4)] / 6=-0.67$ Thus, the negative SMRD represents the tendency in this example to look at the benefits first. 
Time and Fixation Proportion scores: Total inspection time (summing durations across all fixations) and number of fixations were computed for each IA. These measures were then converted to proportions by dividing by total inspection time or number of fixations across both interest areas. Because the percentage proportion scores for risks and benefits sum to $100 \%$, we report only the percentage proportion scores for benefits. Because the correlation between the number of fixations on an area and the time spent fixating was very high across all scenarios ( $r$ 's .89 to .97 ) and the pattern of results consistent for the two measures, only the time proportion measure is reported here. ${ }^{4}$

Number of Transitions: The number of transitions was calculated from the fixation data: with a "transition" defined as each time the participant's gaze switched from attending to benefit information to attending to risk information, or vice versa (i.e., when two successive fixations were on different IAs). If a participant read all the risks first and then read all the benefits without switching back, their transition score would be 1. Each of these eye-tracking measures was calculated separately for each scenario.

\section{$\underline{\text { Data Analysis }}$}

We present the Study 2 eye-tracking data first because (reflecting that information search precedes information processing) this checks for the impact of the presentation order and orientation manipulations. Here, we examined the effect of presentation order and orientation on each gaze measure from study 2 (the eye tracking study). The data were analysed using a Generalised Estimating Equations (GEE) ${ }^{5}$ model with an exchangeable correlation matrix, robust standard errors, and Gaussian identity matrix. This allowed us to collapse across scenarios in the repeated measures design (which randomised participants to orientation and order across the four scenarios). In each analysis in Study 2, 175 responses were analysed (4

\footnotetext{
${ }^{4}$ The data for the inferential analysis conducted on fixation proportion is included in Table 1.

${ }^{5}$ The GEE model represents a flexible approach to handling correlated data structures (e.g. studies with repeated measures from the same individual over time); see Honish, Edwards, Elden \& Leonard[16].
} 
scenarios per participant; apart from one participant whose responses were not recorded for the final scenario due to an eye-tracker malfunction). GEE analysis was also used to examine the relationships between eye-tracking measures and treatment evaluations, with all predictors included in the model. Analyses were conducted using STATA, and we report regression coefficients (B), corresponding z-scores (test statistics) and $p$-values.

Studies 1 and 2 had the same experimental manipulations of order. However, because of the slightly different design and software used in the eyetracking study 2 , allocation of participants and scenarios to conditions differed (counterbalancing vs. randomisation). It was therefore more appropriate to analyse each of the 4 scenarios separately for each study. This was done using factorial ANOVA in SPSS, with order and orientation as factors, and treatment evaluation as the dependent variable. Partial eta-squared $\left(\eta^{2}\right)$ is reported as a measure of effect size, which estimates the proportion of variation accounted for by an effect that is not accounted for by other effects.

\section{Funding Source}

These studies were funded by the $\mathrm{PhD}$ studentship awarded to the first author from the University of Cambridge (Winton Fund) and the UK Economic Social Research Council (ESRC). The funding source had no role in the study topic, design, analysis or reporting.

\section{Results}

\section{Sample Characteristics}

Sample Demographics: Of the 108 who participated in study 1, 99 had completed the prescreen questionnaire. Of this sample, $68(68.7 \%)$ were female and 31 male, with 55\% aged $16-20,39 \%$ aged $21-28$, and $6 \%$ aged over 29 years. Of the 44 participants in study 2, 34 had completed the pre-screen questionnaire. Of these, 21 (61.8\%) were female and 13 male, with $15 \%$ aged $16-20,71 \%$ aged $21-28$, and $15 \%$ aged over 29 years. 


\section{The effect of order and orientation on gaze behaviour: Eye-tracking data from Study 2}

Figure 2 presents the descriptive statistics, and Table 1 the inferential analyses, for each GEE analysis. For each analysis, step 1 (without interaction) and step 2 (including interaction term) is shown. As described in the data analysis section, this analysis was conducted collapsed across scenarios, allowing for a single analysis of the overall effect of order and orientation on these eye-tracking measures to be investigated.

\section{***Figure 2 \& Table $1 * * *$}

Despite the presentation allowing participants to look in whatever order they wanted, their looking order was influenced by table layout (Figure 2a). There was a significant effect of presentation order on the actual search order (SMRD Order score): Participants' search order matched the typical top-left to bottom-right gaze pattern. For those presented with the benefits "first", thus at the top or left of a table, the negative SMRD order score revealed that they typically read the benefit information before the risk information. The average SMRD order scores are closer to the limits of $(+1$ and -1$)$ than a score of 0 . Given that these limits represent cases of strict adherence to the order of presentation, this pattern suggests that reading processed within an information block (e.g., all the benefits) before transitioning to the other set. There was no significant main effect of orientation, however the significant order-by-orientation interaction on SMRD order scores reflects that this within-set search strategy is stronger in the vertical than in the horizontal orientation. Thus, while both t-tests of the simple-main effects of order at each level of orientation were significant $(p<.001)$, the effect was substantially larger in the vertical orientation compared to the horizontal orientation ( $\eta^{2}$ of .955 vs. .768).

This pattern is supported by the analysis of the number of transitions between the risk and benefit blocks (Figure 2b). Consistent with a stronger order effect in the vertical 
Role of order on search and treatment perceptions

orientation, a significant main effect of orientation on the number of transitions was found. Thus, the number of transitions between the risk information and the benefit information was lower in the vertical orientation than horizontal orientation (i.e. there was a significant main effect of orientation). When presented side-by-side (horizontal orientation), switching between the information sets was more frequent. This pattern is consistent with a propensity to make horizontal, rather than vertical, saccades[4] or follow a "z-path" in reading[17]. No main effect of order, or interaction between order and orientation, was found.

When examining the proportion of time spent reading the benefits (Figure 2c), a significant main effect of order was found. Thus, presented with the benefits first, participants generally spent a similar amount of time looking at benefits and risks (balanced: around 50\% for each). However, when the risks were presented first, the amount of time spent on the benefits dropped below $50 \%$ and search was "risk heavy", with approximately $60 \%$ of the time spent looking at the risks. No significant main effect of orientation on time proportion spent reading the benefits, nor any significant interaction between order and orientation, was found.

This relationship between order of presentation and the proportion of time spent on each IA is further illustrated by the negative relationship between SMRD order score and the proportion of time spent on the benefits $(\mathrm{r}(173)=-.36, \mathrm{p}<.001) .{ }^{6}$ When participants looked mainly at the risks first (i.e. a positive SMRD value), they spent less time looking at the benefits. All relationships between the final search measure, number of transitions, and the other search measures were weak (all $|\mathrm{r}|<.072$, all $\mathrm{p}>.341$ ).

\section{Mapping the relationship between search measures and treatment evaluations}

\footnotetext{
${ }^{6}$ This statistic is calculated collapsed across the scenarios. Correlational analysis conducted for each scenario separately can be found in Appendix 3.
} 
Role of order on search and treatment perceptions

Assessing the relationship between the search measures and overall treatment evaluations (collapsed across scenarios), neither SMRD order score $(\mathrm{r}(173)=.04, \mathrm{p}=.618)$ nor number of transitions $(\mathrm{r}(173)=-.02, \mathrm{p}=.809)$ showed a significant correlation with overall treatment evaluations. Time proportion showed a stronger relationship with treatment evaluations, though this effect was not significant; $\mathrm{r}(173)=.14, \mathrm{p}=.060$. However, when controlling for looking order (SMRD order score) and number of transitions between risks and benefits in our model, time proportion was the only significant predictor of treatment evaluations $(B=0.02, z=2.28, p=.023)$. Neither of the other search variables were significant predictors (all $|z|<1.27, p>.204$ ). Thus, holding other variables constant, the proportion of time spent attending to benefits positively (though weakly) predicted treatment evaluations, such that trials where the observer spent more time fixating the benefits led to slightly higher average treatment evaluations.

\section{The effect of manipulated order and orientation on overall treatment evaluations}

Analysis of the assigned order and orientation conditions was deemed appropriate because the eye-tracking data revealed that, generally, the order of presentation and order of processing were consistent. These analyses are summarized in Figures 3 and 4, with the full details of each ANOVA reported in Table 2; because of a difference in condition randomisation (see Data Analyses sub-section), the analysis was conducted separately for each scenario, rather than as a combined analysis.

\section{***Figure $3 \& 4$ and Table2***}

There was no consistent effect of presentation order on treatment evaluation, which is consistent with little relationship between the SMRD score and evaluations already described. Conducting the same analysis collapsed across the treatment scenarios in study 1 , there was no significant effect of orientation $(\mathrm{F}(1,100)=0.002, \mathrm{p}=.969)$, order $(\mathrm{F}(1,100)=0.45$, 
Role of order on search and treatment perceptions

$\mathrm{p}=.504)$ or interaction between order and orientation $(\mathrm{F}(1,100)=0.06, \mathrm{p}=.812)$. All effect sizes were negligible $\left(\eta^{2}<.004\right)$. Therefore, there is little evidence for a direct effect of order or orientation on treatment evaluations across either study.

\section{General Discussion}

Bergus et al.[6] described a recency-based order effect, whereby presenting the risks last led to lower ratings of favourability for an aspirin treatment. Despite choosing scenarios of a similar risk level (i.e. non-invasive, medicinal treatments), our studies found little support for an effect of information order on treatment evaluations. Moreover, our search data points towards a primacy advantage for negative information as the more likely possibility.

Results from our search process measures (eye-tracking) revealed two key sets of findings. First, analysis of reading patterns shows that table layout affects how people attend to a table's contents. Participants usually attended first to the left or top area of the display, irrespective of whether this showed risks or benefits. There was a "match" between presentation layout and looking order, which reflected the reading patterns and gaze biases of the cultures from which most of our participants came[2][3][4]. Even with simultaneous presentations where an individual is free to search in their own order, choices about where to place information still constrain search. Thus, participants tend to read all the information in one information block before transitioning to the other information block: for example, all risks before all benefits. However, the orientation of the table affects the strength of this tendency. Specifically, data from looking order and transitions show that the tendency to read information within a set, and with few gaze transitions between sets, is more pronounced in the vertical orientation than the horizontal orientation. This might influence how patients process risks and benefits. For instance, patients may treat items of information that are 
Role of order on search and treatment perceptions

presented side-by-side as paired, even if this was not the intent of the designer. Hills and Hertwig[18] demonstrated that such "pairwise" sampling of attributes from two options can be associated with different preferences compared to when all attributes of one option are considered before all the attributes of the other option. Simply transposing the rows and columns of a table could affect treatment preferences.

Second, we found that where risk and benefit information is placed within a table affected the time spent on reading the benefits. When benefits were presented on the left or top of the display, $50 \%$ of the time was spent on risks. In contrast, around $60 \%$ of the time was spent on the risks when the risks were shown to the left or top of the table. Thus, the risk information appears to be more attention "grabbing" when presented where it will usually be read first. Participants may have found it difficult to disengage from the risk information perhaps reflecting a bias to attend more to negative information[19] - and consequently spent less time on the benefit information. This is something that decision/communication aid designers should be aware of - not least because we found that time proportion spent on the benefits significantly predicted treatment evaluations when holding the other variables constant.

Notwithstanding the potential impact of table layout (e.g., the order and orientation of information) upon information processing, discussed above, we did not find the order effect on preference that we expected to - namely, a recency advantage to information that is read last. We suggest three factors that might account for this discrepancy with previous studies who do report such an effect. First, in our scenarios, we matched the list of risks and the list of benefits for their number of items, their word count and character count. This does not 
seem to be the case in Bergus et al.'s[6] aspirin scenario where the risk list was longer ${ }^{7}$. This may have enhanced any order effects in previous studies.

Second, for short item-lists (2-12 items), Hogarth and Einhorn reported that task differences predict whether, and in what direction, order effects are found in evaluation studies [20]. Recency effects are more common when information is evaluated via a step-bystep response procedure, whereby participants express their beliefs after encountering each piece of information. Primacy effects are more common when information is evaluated via an end-of-sequence procedure, in which participants report opinions only after all information is presented. While not specifically requesting a response after each item of information, Bergus et al.[6] asked participants for their initial opinion of the treatment. By providing such a starting anchor, this may have encouraged participants to adopt a step-by-step processing strategy. In contrast, our studies required only a response after viewing all information (an end-of sequence procedure), so no initial anchor value was provided or self-generated. With no pre-specified anchor, it is the first piece of information which often acts as an anchor for subsequent comparison[20]. This leads to mid-scale evaluations in both orders and only weak order effects (as seen in our data). Figure 5 illustrates how, according to Hogarth and Einhorn's[20] analysis, these differences might lead to different patterns of results.

\section{$* * *$ Figure $5 * * *$}

Third, with only three risks and three benefits, it may have been straightforward for participants to hold all 6 pieces of information coded as separate chunks in memory[21] - at least once these items had been processed for their 'gist' meaning[22]. If participants were able to assess all pieces of information simultaneously, this may have attenuated any possible order effects.

\footnotetext{
${ }^{7}$ For the aspirin scenario, Bergus et al.[6] presented one sentence of benefit information and four different side effects. We presented three of those four risks alongside three identified benefits.
} 


\section{Limitations}

With little numerical information, it was not our belief that numeracy would play a key role in participants' search or evaluations, however traditionally in risk communication research, a feature of university students, which may limit generalizability, is that such participants are usually highly numerate[23]. Potentially reducing the impact of this limitation on this study, previous investigations recruited from the sample participant pool (i.e., University of Essex Students) reveal a bias towards lower levels of numeracy compared to general population norms (see Heard, Rakow \& Spiegelhalter[24]) ${ }^{8}$. As with any university sample, who are associated with more years of education, it is likely they have had considerable practice at reading. Thus, what we have observed might not hold for all levels of reading ability.

We chose scenarios that were relatively simple (i.e., one treatment option with a few risks and benefits presented); therefore, these results cannot provide clear evidence for what happens when more complex scenarios are presented. However, our search findings provide a basis for some predictions for such complex scenarios, which future research should investigate.

Finally, we chose to control the risk and benefit list length. This was necessary to avoid list length being confounded with information type (risk vs. benefit). Without this, for example, the bias in search towards risk information (when presented first) that we observed could, instead, be attributed to message length. While an important control to understand the effects in theory and providing a foundation for predictions in more diverse scenarios - more naturalistic designs, with a higher proportion of risks than benefits may alter the pattern of information search.

\section{Future Directions}

\footnotetext{
${ }^{8}$ This likely reflects that the University has very few students studying physical sciences or engineering.
} 
These findings highlight how order and orientation affect information search, including: more "risk heavy" search when risks are presented first, and effects arising from the tendency to make horizontal rather than vertical saccades. The impact of these effects might become more pronounced as scenarios become more complex and comprise more information. Such scenarios are common in healthcare and medical decisions, particularly when choices involve multiple options and/or attributes (e.g. NHS Choices[25]).

For two reasons, we expect that information order will have greater impact on patient preference for complex choices than we saw in our simple treatment scenarios. First, Hogarth and Einhorn[20] report that primacy effects are common for long lists of information, irrespective of whether evaluations are made step-by-step or at the end of the sequence or presentation. Second, because attention wanes in lengthy decision tasks[26], we anticipate that information order will have a greater effect on the time proportion spent on benefits in complex tasks. For example, when risks are presented first, search through a large table should be even more "risk heavy" than we observed for small tables of information. In turn, this should increase the chance that table layout will affect preference.

For multi-attribute and multi-option choices, our finding that table layout affects the rate of switching between information sets suggests a further possibility. We predict that reduced switching in the vertical orientation may have implications for how treatment options are compared when two or more options are presented in a tabular format: either side-by-side, or above-and-below. With above-and-below presentation, we expect relatively more withinoption processing (consistent with forming a global opinion of each option based on attribute information). This should occur because each row displays the attributes for a given option, and so when gaze "tracks" along a row a single option is being processed. Conversely, for side-by-side presentation, we expect relatively more comparison between options within a given attribute. This should occur because different rows represent different attributes, and 
so typical gaze patterns with many horizontal saccades encourage comparing options for a given attribute. Those different search strategies lend themselves to adopting different strategies for choice that can alter which option is preferred[12][18][27].

\section{Conclusion}

Our eye-tracking data show how presentation order and table orientation affect information acquisition. This highlights the importance of seemingly arbitrary choice-architecture design features (e.g., the layout of information in a table). In particular, we identify two noteworthy search-processing effects. First, the tendency to read information within sets or in information blocks (e.g., read all of the risks and then all of the benefits) is stronger when sets are presented in an above-below orientation rather than a side-by-side orientation. Second, information search is more risk-heavy when risks are presented at the top or to the left of the display. It may be that task differences (e.g. response mode) weigh more heavily on subsequent preferences than order and orientation when scenarios are short: This is a possible reason why we did not find clear effects of order and orientation upon treatment evaluations. However, this may not always be so; and we have good reason to expect that the effects of table layout may become more pronounced in more complex medical scenarios. This represents an opportunity for future research. 


\section{References}

[1] NHS Choices. Should I have a PSA test? 2015, March 25 [Online Article] [Accessed 2016 August 27]. http://www.nhs.uk/Livewell/Prostatehealth/Pages/psa-test.aspx.

[2] Foulsham T, Gray A, Nasiopoulos E, Kingstone A. Leftward biases in picture scanning and line bisection: a gaze-contingent window study. Vision Research. 2013;78:14-25

[3] Nielsen J. Horizontal attention leans left. 2010 April 6. [Online Article]. [Accessed 2016 September 27]. https://www.nngroup.com/articles/horizontal-attention-leans-left/.

[4] Foulsham T, Kingstone A, Underwood G. Turning the world around: Patterns in saccade direction vary with picture orientation. Vision Research. 2008;48:1777-1790.

[5] Reber, A.S. The penguin dictionary of psychology ( $2^{\text {nd }}$ edition). England: Penguin Books; 1995, Entry: Primacy effect; p. 594.

[6] Bergus GR, Levin IP, Elstein AS. Presenting risks and benefits to patients. Journal of General Internal Medicine. 2002; 17(8): 612-517.

[7] Ubel PA., Smith DM., Zikmund-Fisher BJ., Derry HA., McClure J., Stark A., Wiese C., Greene S., Jankovic A. Testing whether decision aids introduce cognitive biases: results of a randomised trial, Patient Education and Counseling. 2010;80:158-163.

[8] Rakow T, Demes KA, Newell BR. Biased samples not mode of presentation: reexamining the apparent underweighting of rare events in experience-based choice. Organizational Behavior and Human Decision Processes. 2008;106(2):168-179. 
Role of order on search and treatment perceptions

[9] Rakow T, Newell BR. Degrees of uncertainty: An overview and framework for future research on experience-based choice. Journal of Behavioural Decision Making. 2010;23:1-14.

[10] Ashby NJS, Rakow T. Forgetting the past: individual differences in recency in subjective valuations from experience. Journal of Experimental Psychology: Learning, Memory, \& Cognition. 2014;40:1153-1162.

[11] Pachur T, Hertwig R, Wolkewitz R. The affect gap in risk choice: affect-rich outcomes attenuate attention to probability information. Decision. 2013;1(1):64-78.

[12] Glöckner A, Herbold A-K. An eye-tracking study on information processing in risky decisions: evidence for compensatory strategies based on automatic processes. Journal of Behavioural Decision Making. 2011;24 (1):71-98.

[13] Cokely ET, Galesic M, Schulz E, Ghazal S., Garcia-Retamero R. Measuring risk literacy: the Berlin numeracy test, Judgment and Decision Making. 2012;7(1):25-47.

[14] Johnson EJ, Häubl G, Keinan A. Aspects of endowment: A query theory of value construction, Journal of Experimental Psychology: Learning, Memory \& Cognition. 2007;33(3):461-47.

[15] Hardisty, D., Johnson, EJ, Weber, EU. A dirty word or a dirty world? Attribute framing, political affiliation and query theory. Psychological Science, 2010;21(1):86-92.

[16] Honish, GG, Edwards, EP, Eiden, RD, Leonard, KE. Analysing family data: A GEE approach for substance use researchers, Addictive Behaviours, 2010;35(6):558-563.

[17] Cohn N. Navigating comics: An empirical and theoretical approach to strategies of 
reading comic page layouts. Frontiers in Psychology, 2013;4:1-15.

[18] Hills TT, Hertwig R. Information search in decisions from experience: Do our patterns of sampling foreshadow our decisions? Psychological Science, 2010;21(12):1787-1792.

[19] Vaish A., Grossmann T., Woodward, A. Not all emotions are created equal: The negativity bias in social-emotional development. Psychological Bulletin, 2008;134(3):383-403.

[20] Hogarth RM, Einhorn HJ. Order effects in belief updating: The belief-adjustment model. Cognitive Psychology, 1992; 24(1): 1-55.

[21] Miller GA. The magic number seven, plus or minus two: Some limits on our capacity for processing information. Psychological Review, 1955;101(2):343-352.

[22] Reyna VF. A theory of medical decision making and health: Fuzzy trace theory. Medical Decision Making, 2008;28(6):850-865.

[23] Peters, E, Västfjall D, Slovic P, Metrz, CK, Mazzocco, K, Dickert, S. Numeracy and decision making, Psychological Science, 2006;17: 407-413.

[24] Heard, C.L., Rakow, T., \& Spiegelhalter, D. (2018). Using Speed-of-Ageing Metaphors in Health Communication: A comparison of the comprehension and perception for speed-of-ageing and hazard ratio formats. Applied Cognitive Psychology, 32, 81-93.

[25] NHS Choices. Services near you: Results for hospitals in London, 2017. [Webpage]. [Accessed 2017 February 1]. http://www.nhs.uk/servicesearch/Hospital/London/Results/3/-0.085/51.511/7/13136?distance=25

[26] Ashby NJS, Rakow T. Eyes on the prize? Evidence of diminishing attention to 
Role of order on search and treatment perceptions

experienced and foregone outcomes in repeated experiential choice. Journal of Behavioral Decision Making. 2016;29(2-3):183-193.

[27] Payne JW, Bettman J., \& Johnson, EJ. The adaptive decision maker. Cambridge: Cambridge University Press; 1993. 


\section{TABLES}

Table 1: Descriptive and inferential statistics for each order $\mathrm{x}$ orientation (on search measures) analysis (Study 2)

\begin{tabular}{|c|c|c|c|c|c|c|c|c|c|c|}
\hline \multirow[t]{2}{*}{ Analysis } & & \multicolumn{3}{|c|}{$\begin{array}{l}\text { Main Effect } \\
\text { Orientation }\end{array}$} & \multicolumn{3}{|c|}{ Main Effect Order } & \multicolumn{3}{|c|}{$\begin{array}{c}\text { Orientation-by-Order } \\
\text { Interaction }\end{array}$} \\
\hline & & B & $\mathrm{Z}$ & $\mathrm{p}$ & $\mathrm{B}$ & $\mathrm{Z}$ & $\mathrm{p}$ & B & $\mathrm{Z}$ & $\mathrm{p}$ \\
\hline \multirow{2}{*}{$\begin{array}{l}\text { SMRD Order Score } \\
\text { (Note: Score of } 1 \text { denotes reading all } \\
\text { risks before all benefits, while -1 } \\
\text { denotes reading all benefits before } \\
\text { all risks). } \\
\end{array}$} & STEP 1* & -0.017 & -0.61 & .545 & 0.81 & 26.49 & $<.001$ & -- & -- & -- \\
\hline & STEP 2** & -0.012 & -0.47 & .639 & 0.80 & 24.44 & $<.001$ & 0.08 & 2.59 & .009 \\
\hline \multirow[t]{2}{*}{ Number of Transitions } & STEP 1 & -0.83 & -4.06 & $<.001$ & 0.24 & 1.34 & .179 & -- & -- & -- \\
\hline & STEP 2 & -0.85 & -4.09 & $<.001$ & 0.27 & 1.41 & 0.157 & -0.20 & -1.29 & .196 \\
\hline \multirow{2}{*}{$\begin{array}{l}\text { Time Proportion } \\
\text { (On Benefits) }\end{array}$} & STEP 1 & -0.58 & -0.84 & .399 & -4.43 & -4.82 & $<.001$ & -- & -- & -- \\
\hline & STEP 2 & -0.57 & -0.82 & .411 & -4.52 & -4.88 & $<.001$ & 0.52 & 0.63 & 0.531 \\
\hline \multirow{2}{*}{$\begin{array}{c}\text { Fixation Proportion } \\
\text { (On Benefits) }\end{array}$} & STEP 1 & -0.59 & -0.98 & .326 & -4.55 & -5.40 & $<.001$ & -- & -- & -- \\
\hline & STEP 2 & -0.85 & -0.90 & .366 & -4.86 & -4.08 & $<.001$ & 1.05 & 0.35 & 0.725 \\
\hline
\end{tabular}




\begin{tabular}{|c|c|c|c|c|c|c|c|c|c|c|}
\hline \multicolumn{11}{|c|}{$\begin{array}{c}\text { Table 2: inferential Statistics for the Effect of Order and Orientation on Overall } \\
\text { Treatment Evaluations }\end{array}$} \\
\hline \multirow[t]{2}{*}{ Scenario } & \multirow[b]{2}{*}{$\begin{array}{l}\text { Study } \\
\left(d f_{1}, d f_{2}\right)\end{array}$} & \multicolumn{3}{|c|}{$\begin{array}{l}\text { Main Effect } \\
\text { Orientation }\end{array}$} & \multicolumn{3}{|c|}{ Main Effect Order } & \multicolumn{3}{|c|}{ Interaction } \\
\hline & & $\mathrm{F}$ & $\mathrm{p}$ & $\eta_{\mathrm{p}}^{2}$ & $\mathrm{~F}$ & $\mathrm{p}$ & $\eta_{\mathrm{p}}^{2}$ & $\mathrm{~F}$ & $\mathrm{p}$ & $\eta_{\mathrm{p}}^{2}$ \\
\hline \multirow[t]{2}{*}{ Aspirin } & $\begin{array}{c}1 \\
(1,104)\end{array}$ & 2.27 & .135 & .021 & 1.30 & .257 & .012 & 0.95 & .333 & .009 \\
\hline & $\begin{array}{c}2 \\
(1,40) \\
\end{array}$ & 0.18 & .673 & .005 & .071 & .403 & .018 & 0.60 & .443 & .015 \\
\hline \multirow{2}{*}{ Anticoagulants } & $\begin{array}{c}1 \\
(1,101)\end{array}$ & 1.83 & .179 & .018 & 0.08 & .780 & .001 & 0.20 & .657 & .002 \\
\hline & $\begin{array}{c}2 \\
(1,40)\end{array}$ & 0.11 & .744 & .003 & 0.42 & .519 & .010 & $<.001$ & .999 & $<.001$ \\
\hline \multirow{2}{*}{ ACE Inhibitors } & $\begin{array}{c}1 \\
(1,102)\end{array}$ & 0.56 & .457 & .005 & 7.90 & .006 & .072 & 0.01 & .922 & $<.001$ \\
\hline & $\begin{array}{c}2 \\
(1,39)\end{array}$ & 0.90 & .349 & .023 & 0.33 & .571 & .008 & 1.96 & .170 & .048 \\
\hline Statins & $\begin{array}{c}\mathbf{1} \\
(1,101)\end{array}$ & 1.10 & .298 & .011 & 0.20 & .653 & .002 & 1.34 & .250 & .009 \\
\hline
\end{tabular}




\section{FIGURES}

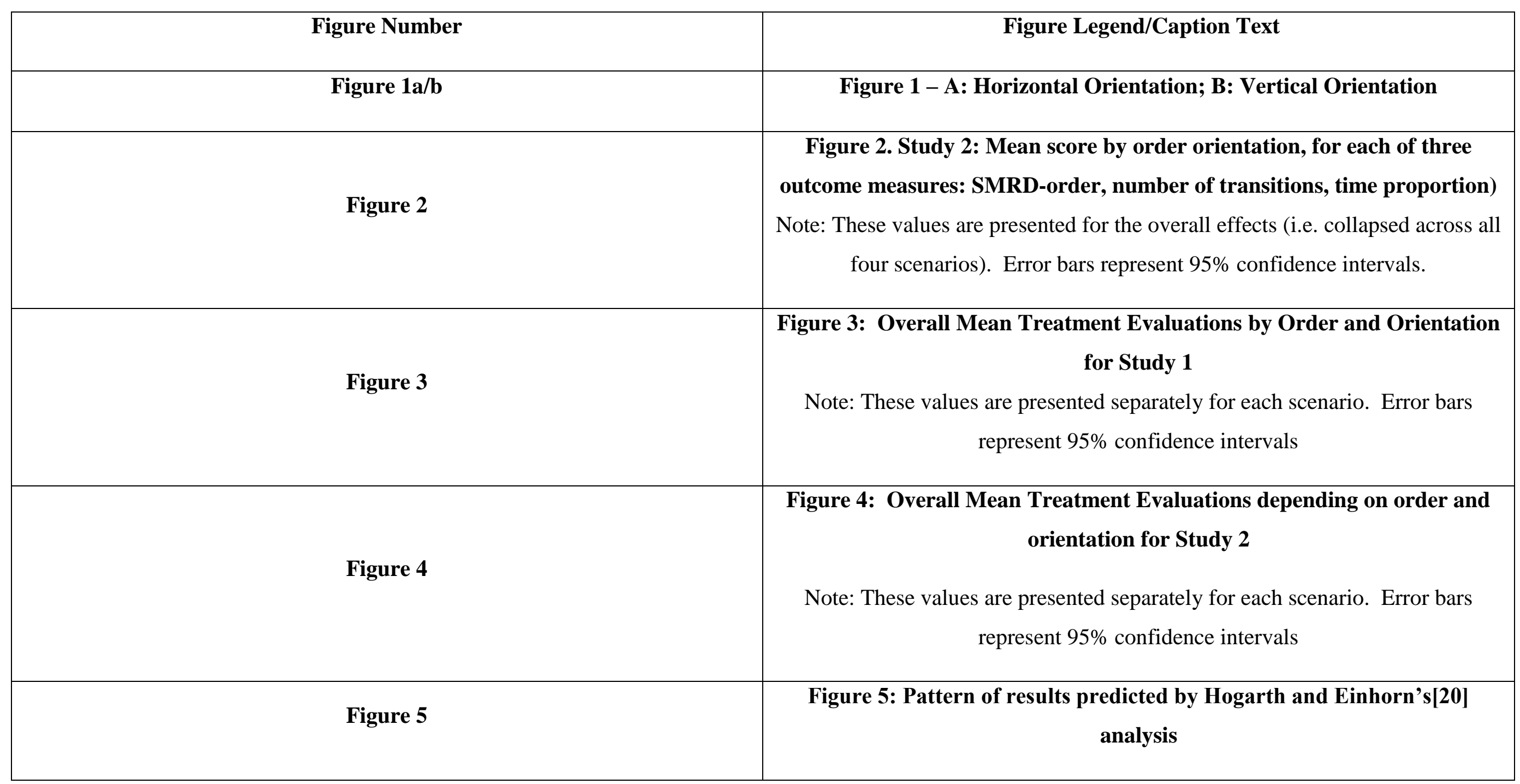




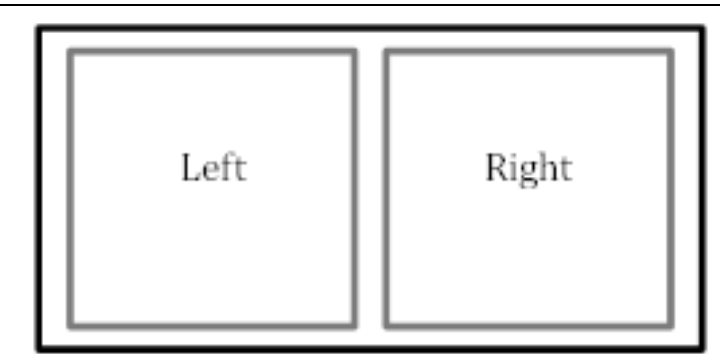

A: Horizontal Orientation

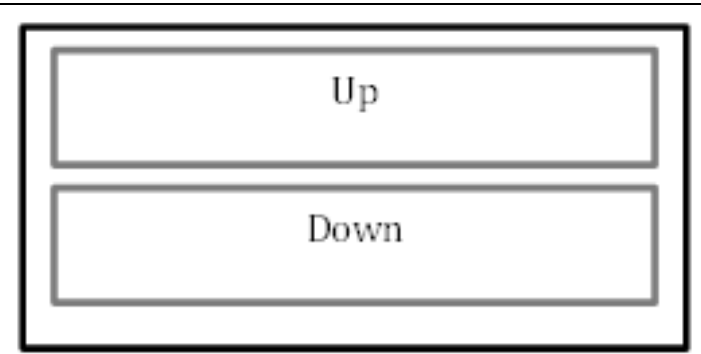

B: Vertical Orientation

Figure 1 - A: Horizontal Orientation; B: Vertical Orientation 


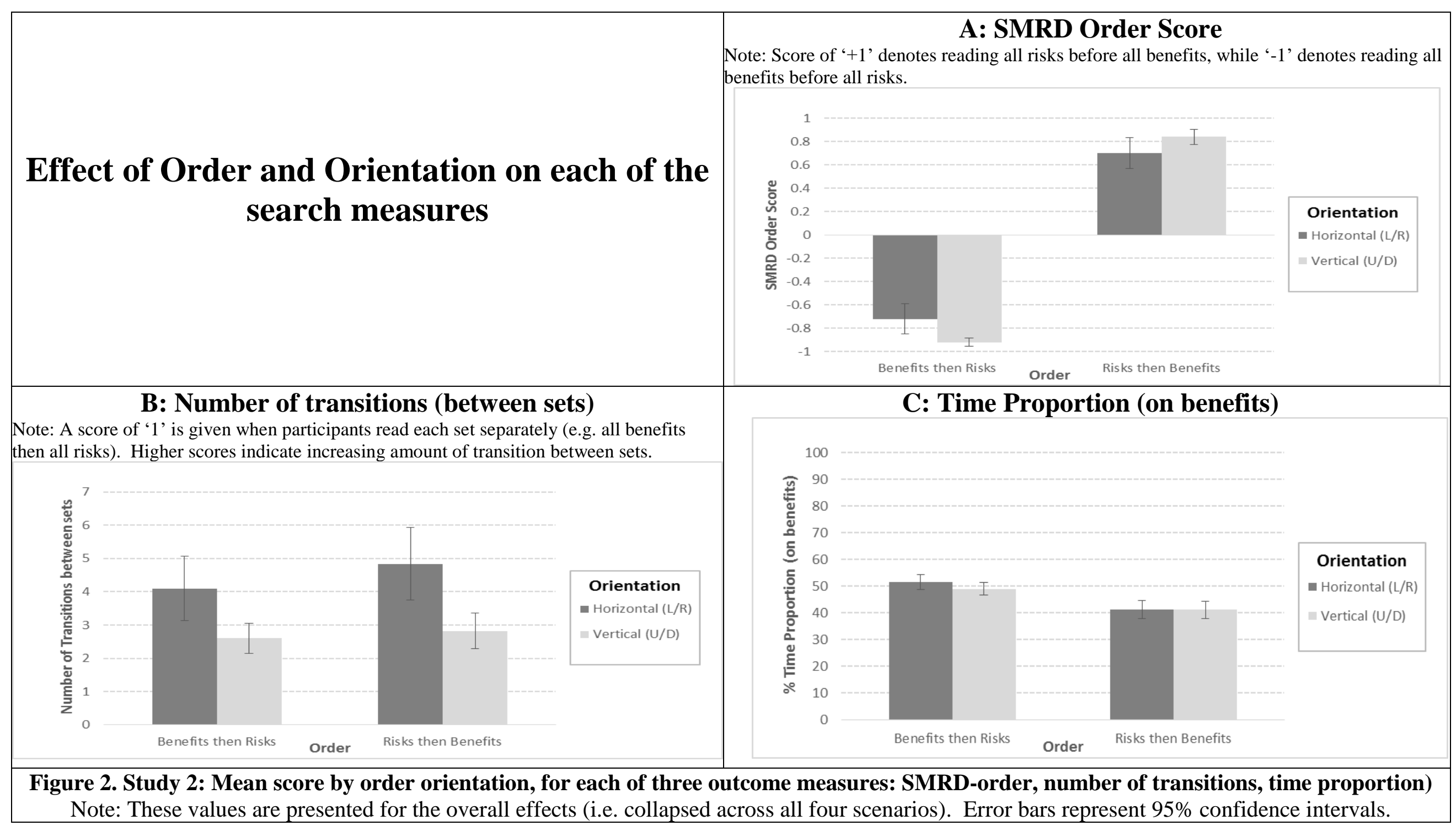




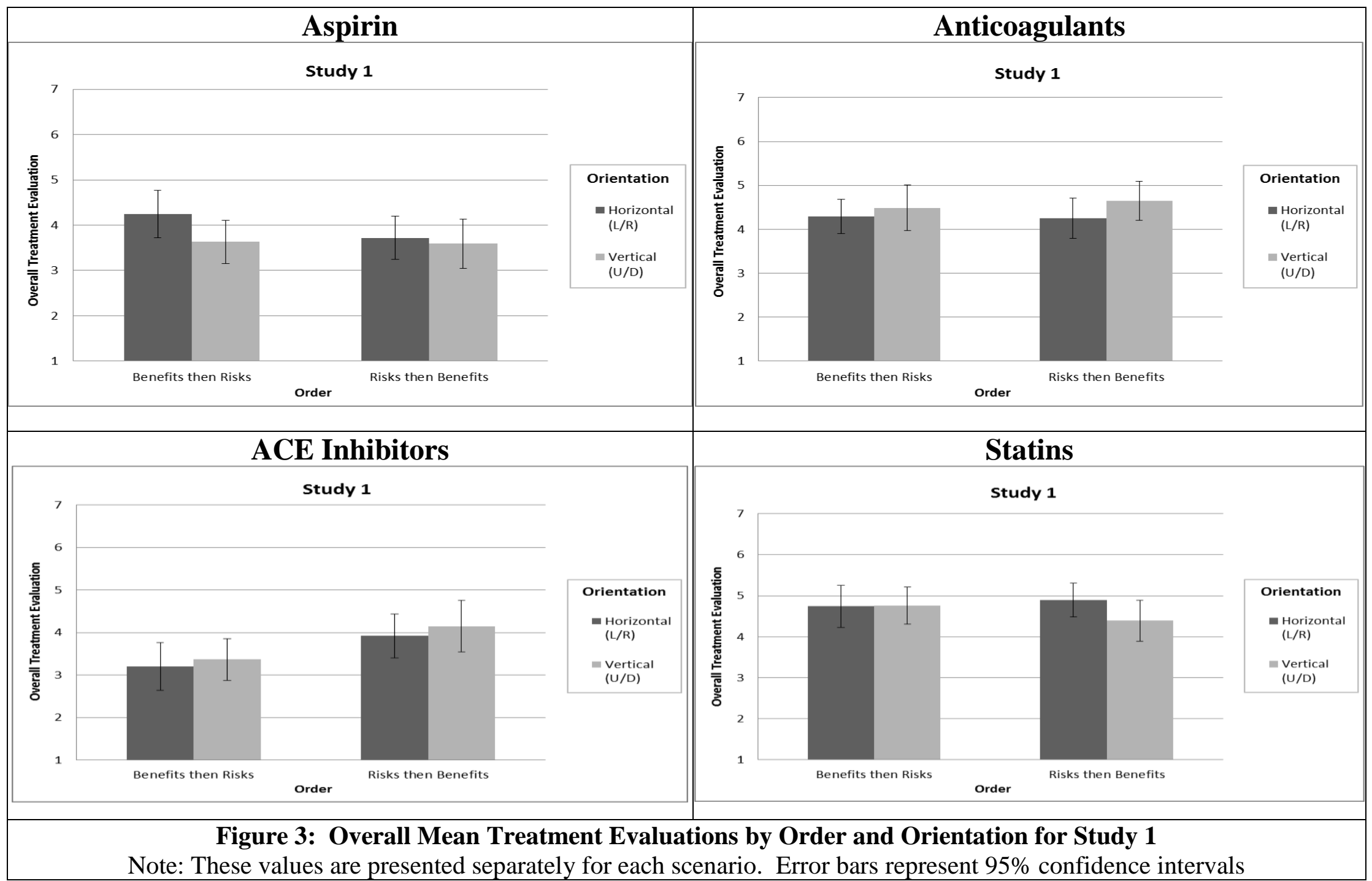


Role of order on search and treatment perceptions

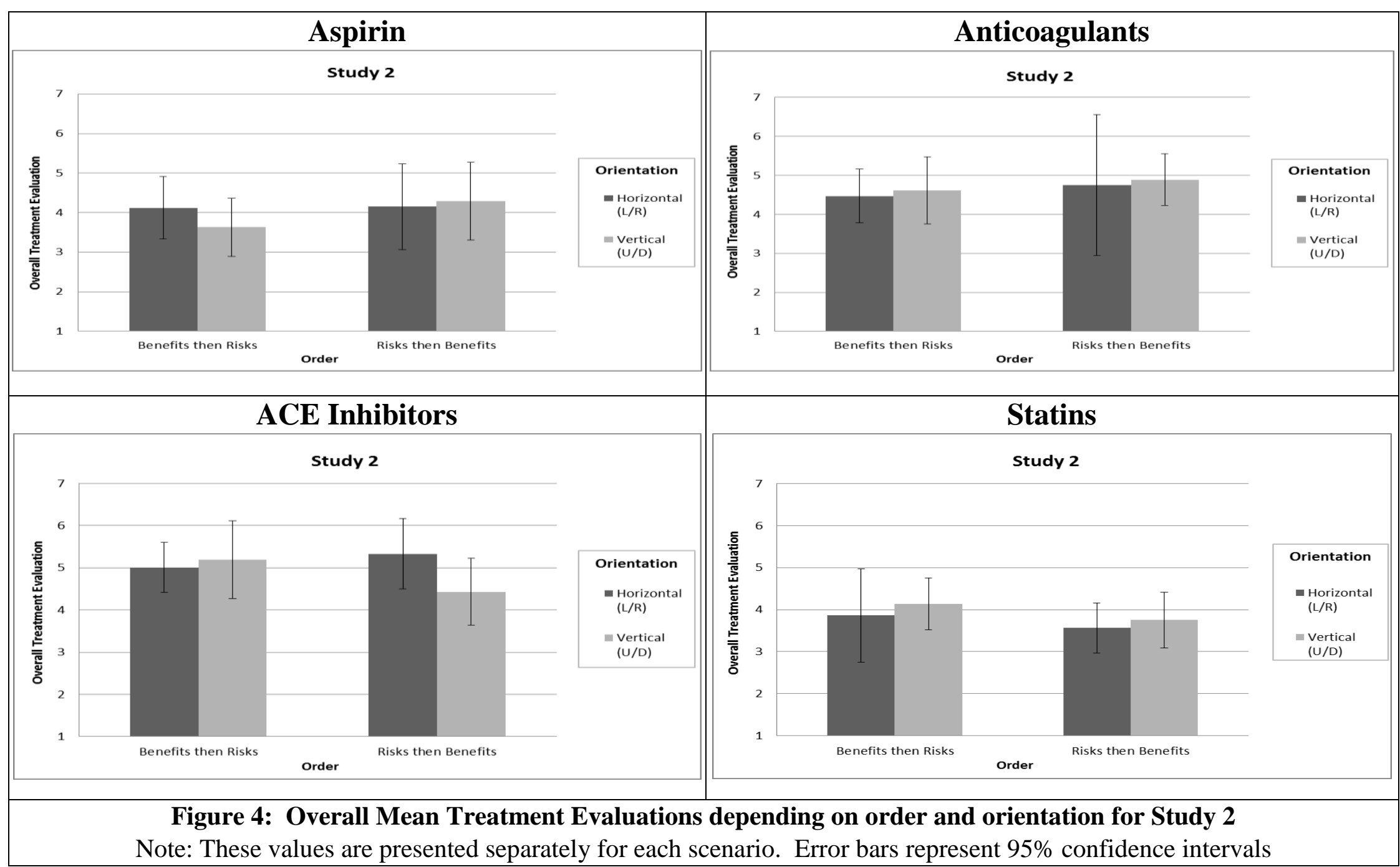




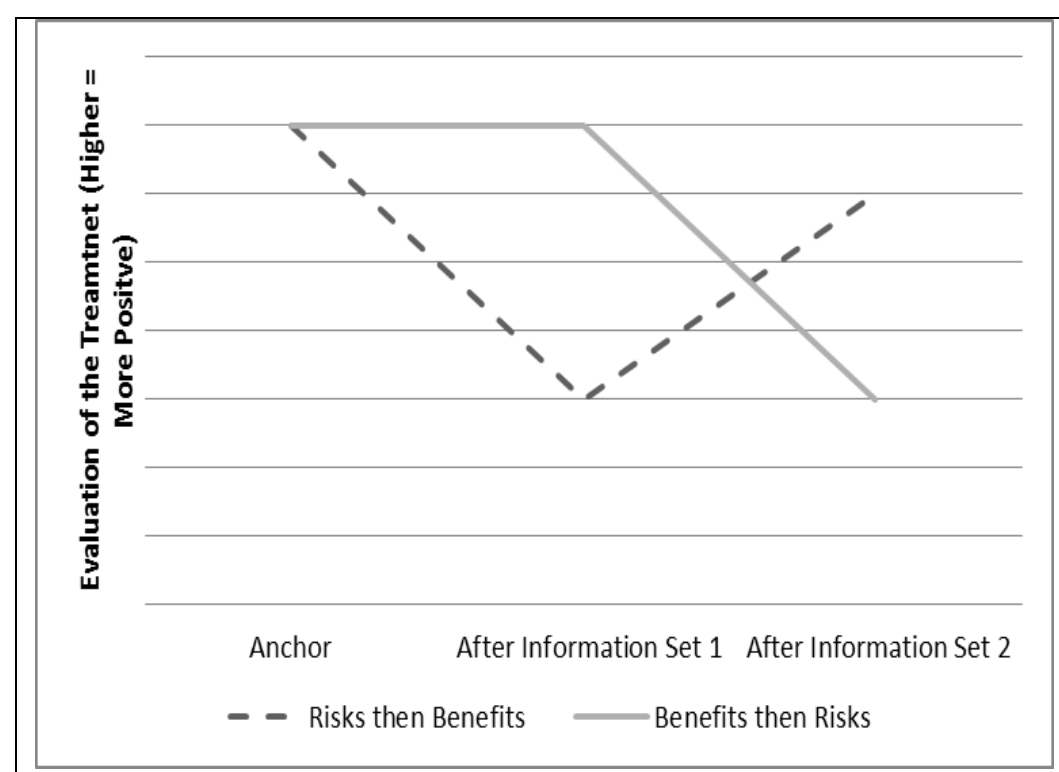

A

Bergus et al.[6]

with initial anchor judgement

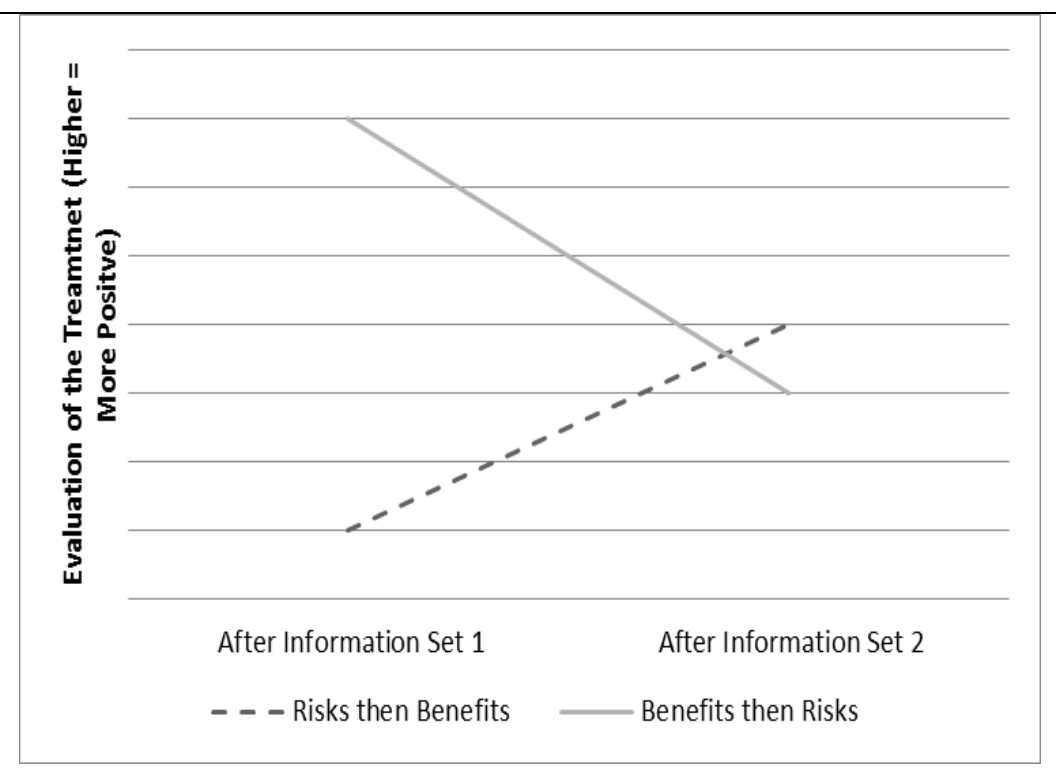

B

Current Experiment

No Initial anchor judgement

Figure 5: Pattern of results predicted by Hogarth and Einhorn's[20] analysis 


\section{$\underline{\text { Appendices }}$}

Appendix 1: Examples of the information presented scenario background and risk and benefit pages. For ease, all scenarios are shown presented in the Horizontal (left/right) Benefits then Risks (LRBR) order.

\section{Aspirin:}

Imagine that you have experienced an episode of numbness of the face and right arm. You go to your doctor and after running a few tests; your doctor tells you that you have mild carotid stenosis.

You doctor explains that carotid stenosis is a narrowing or blockage of the carotid arteries. The carotid arteries are the two large blood vessels in your neck, which carry oxygen-rich blood to your brain. Carotid stenosis is a common cause of stroke.

One of the recommended treatment options is to take aspirin.

\section{Benefits}

Non Invasive.

For some individuals, aspirin therapy may also reduce the risk of a heart attack.

With aspirin therapy, the risk of stroke is decreased by half. Thus, a reduction from $2 \%$ to $1 \%$ risk of stroke over three years.

\section{Risks}

There is a $40 \%$ higher rate of stomach/ intestinal problems, including a 2 -fold increase in the risk of ulcers. problems.

Aspirin users are twice as prone to experience an intracerebral hemorrhage (burst blood vessel in the brain) 


\section{Anticoagulant:}

Imagine that you have been feeling pain in your right leg, and it has swollen in size. You visit your doctor, who explains that you have deep vein thrombosis.

Deep vein thrombosis is when a blood clot forms within one of your leg veins, which runs through the muscles of the calf and the thigh. In some cases, deep vein thrombosis can lead to further complications such as pulmonary embolism (blood clot in one's lung) or a stroke.

One of the recommended treatment options is to take anticoagulant medicine (e.g. warfarin) to stop the clot getting

bigger and help reduce the ability of the blood to clot.

\section{Benefits}

It reduces the likelihood of recurrence of deep vein thrombosis.

It reduces the likelihood of further complications such as a pulmonary embolism (blood clots in lungs) or stroke.

If serious bleeding occurs or surgery for another health condition is needed, the blood-thinning effects of warfarin can be reversed quickly.

\section{Risks}

There is an increased risk of bleeding problems, both minor (nose) and major (stomach). Approximately $2 \%$ of patients experience a major bleed in the first 3 months of therapy.

Anticoagulant treatments (e.g. warfarin) can require regular blood testing hospital appointments.

Will bruise more easily and cuts take long to heal. 
ACE inhibitors:

Imagine that you visit your doctor for a routine check-up. During this check-up your doctor measures your blood pressure and explain that you have high blood pressure (hypertension).

High blood pressure (or hypertension) is associated with an increased risk of cardiovascular disease such as heart attack or stroke.

One recommended treatment option is to take ACE (Angiotensin Converting Enzyme) inhibitors, which lower one's blood pressure.

\section{Benefits}

ACE inhibitors help to reduce blood pressure level.

Reduce the risk of cardiovascular diseases such as heart attack or stroke.

For those with high blood pressure, reducing it can help these individuals live longer.
Risks

One common side effect is dizziness.

Can cause a long lasting dry cough in around 10 in 100 people who take them.

Less common side effects include swelling of the lips, eyes or tongue and a decline in kidney function. 
Statins:

Imagine you have recently had a blood test. Your doctor has called you in to discuss the results and tells you that you have high cholesterol.

High cholesterol is associated with an increased risk of atherosclerosis (narrowing of the arteries) and coronary heart disease. Additionally, it is associated with other blood flow related health risks such as a heart attack or stroke.

One of the recommended treatment options is to take statins, which lower the level of cholesterol in the blood.

\section{Benefits}

Statins lower the level of cholesterol in your blood.

Statins reduce the risk of developing heart disease and experiencing a heart attack or stroke.

Some research has shown that statins may reduce the risk of developing throat cancer.

\section{Risks}

One common side effect of statin use is muscle pain including soreness or tiredness of muscles.

Statins can cause digestive problems such as nausea, diarrhea or constipation. In rare cases, statins can cause liver damage.

Statin users have an increased risk of Type 2 diabetes. 
Role of order on search and treatment perceptions

Appendix 2: Examples of the six treatment evaluation questions with Likert scale endpoints presented in parentheses

Positively phrased:

(1) How favourable is the treatment? (very unfavourable to very favourable)

(2) Would you choose this treatment? (definitely no to definitely yes)

(3) Would you recommend this treatment? (definitely no to definitely yes)

Negatively phrased:

(4) If you were to choose this treatment, how likely do you think it is that you would experience one of its side effects? (very unlikely to very likely)

(5) Would you avoid this treatment? (definitely no to definitely yes)

(6) How concerned would you be about the side effects? (not concerned to very concerned). 
Appendix 3: Correlational analysis between search measures for each scenario separately

\begin{tabular}{|c|c|c|c|c|c|c|c|}
\hline \multicolumn{8}{|c|}{$\begin{array}{l}\text { Table 1: Correlations between the different process measures and } \\
\text { correlations between overall treatment evaluations and these process } \\
\text { measures for each of the four scenarios. }\end{array}$} \\
\hline $\begin{array}{l}\text { Aspirin } \quad \underline{\text { Statin }} \\
\underline{\text { ACE }} \underline{\text { Anticoagulant }}\end{array}$ & $\begin{array}{l}\text { SMRD } \\
\text { Order } \\
\text { Score }\end{array}$ & $\begin{array}{l}\text { Time } \\
\text { Prop } \\
\text { (on } \\
\text { Bene }\end{array}$ & tion & $\begin{array}{l}\text { Fixat } \\
\text { Prop } \\
\text { (on } \\
\text { Bene }\end{array}$ & ion & $\begin{array}{l}\text { No. of } \\
\text { Transi }\end{array}$ & ons \\
\hline SMRD Order Score & & $\begin{array}{l}-.27 \\
-.49\end{array}$ & $\begin{array}{l}-.51 \\
-.24\end{array}$ & $\begin{array}{l}-.23 \\
-.52\end{array}$ & $\begin{array}{l}-.48 \\
-.31\end{array}$ & $\begin{array}{l}.06 \\
.08\end{array}$ & $\begin{array}{l}.12 \\
.07\end{array}$ \\
\hline $\begin{array}{l}\text { Time Proportion (on } \\
\text { Benefits) }\end{array}$ & & & & $\begin{array}{l}.89 \\
.95\end{array}$ & .97 & $\begin{array}{r}-.10 \\
.05\end{array}$ & $\begin{array}{l}-.12 \\
-.08\end{array}$ \\
\hline $\begin{array}{l}\text { Fixation Proportion } \\
\text { (on Benefits) }\end{array}$ & & & & & & $\begin{array}{r}-.07 \\
.05\end{array}$ & $\begin{array}{l}-.11 \\
-.09\end{array}$ \\
\hline No. of Transitions & \multicolumn{7}{|c|}{$\begin{array}{l}\text { Values reported in this table represent } r \text {-values } \\
\text { Bold }=p<.05, \text { Bold }+ \text { Italics }=p<.001\end{array}$} \\
\hline
\end{tabular}

